\title{
Amplitude modulation of solar p-modes by surface magnetic fields
}

\author{
R. Jain ${ }^{1}$ and C. D. C. Steele ${ }^{2}$ \\ 1 Applied Mathematics Department, University of Sheffield, Sheffield, S3 7RH, UK \\ e-mail: R.Jain@sheffield.ac.uk \\ 2 School of Mathematics, University of Manchester, Manchester, UK
}

Received 3 March 2007 / Accepted 9 July 2007

\section{ABSTRACT}

\begin{abstract}
Context. It is known from Doppler velocity measurements that the amplitudes of solar p-modes are modulated by strong photospheric magnetic field.

Aims. The aim of this paper is to investigate amplitude modulation by model surface magnetic fields.

Methods. Linearised magnetohydrodynamics equations, in the absence of gravity, are used to derive the inhomogeneous wave equation which is then solved using the Born Approximation.

Results. The amount of modulation depends on the plasma beta, the distance from the magnetic region and the wavenumber. It is also found that the direction of observation could also have an effect on the amount of modulation. Finally, the applicability of the findings to the observational data suggests that the modulation depends on the properties of the magnetic field region and measuring it is an un-contaminating probe for the magnetic field.
\end{abstract}

Key words. Sun: magnetic fields - Sun: oscillations - magnetohydrodynamics (MHD) - Sun: helioseismology

\section{Introduction}

Global Helioseismology uses the observed p-modes (acoustic modes) at the Sun's surface to infer the Sun's internal structure (see e.g. Christensen-Dalsgaard 2002). The oscillation parameters such as frequencies, amplitudes and widths are obtained by using peak-fitting routines on Fourier transformed Doppler velocity data and are used for determining internal structure and the dynamics of the Sun's interior (e.g. Gizon \& Birch 2005). To make correct inferences about the solar structure and dynamics, it is essential to estimate the mode frequencies very accurately. However, only part of the whole Sun can be seen at any one time. Also, the line-of-sight measurements such as the Doppler velocity measurements are not efficient at the solar limb where there is more contribution from large scale horizontal motions instead of radial p-modes.

Frequency and amplitude modulation of p-modes in some areas of the solar surface can also cause spatial leakage in the mode spectra. Since the 1980's, it has been realised that the amplitudes and frequencies of solar p-modes are significantly affected by surface magnetic fields.

The modulation of amplitude of p-modes by magnetic regions has been studied by many (e.g. Abdelatif et al. 1986; Braun et al. 1987, 1988; Braun 1995; Hindman \& Brown 1998; Thomas \& Stanchfield 2000; Jain \& Haber 2002; Nicholson et al. 2004). It is now well established that the amplitudes of p-modes are reduced in the frequency band $2-5 \mathrm{mHz}$ and the amount of reduction is a function of frequency and field strength. Various theoretical reasons to explain amplitude reduction are suggested in Jain et al. (1996; see also, Hindman et al. 1997) and Cally et al. (2003).

The effect of magnetic fields on the frequencies of $\mathrm{p}$ modes is also well known (e.g. Woodard \& Noyes 1985; Libbrecht \& Woodard 1990; Howe et al. 1999; Antia et al. 2001). The frequencies of p-modes $(<5 \mathrm{mHz})$ increase with an increase in the magnetic activity during solar cycle and the increase is larger for frequencies of higher degree p-modes. Theoretical reasons for frequency shifts have been investigated by Goldreich et al. (1991) and Jain \& Roberts (1993, 1994, and references therein).

Thus, the spatial and temporal effects of magnetic fields on p-modes are established beyond doubt. To understand these effects in a way that can be described quantitatively would be very useful for reducing any systematic errors that they may cause in the inferred parameters of the Sun's interior. The biggest obstacle to an understanding of the effects of magnetism on p-modes is that the magnetic field structures cannot be directly observed and alternative methods are sought to probe these structures. Various local helioseismology techniques have been developed in recent years (Hill 1988; Duvall Jr et al. 1993; see also, Gizon $\&$ Birch 2005) whose main objective is to infer the sub-surface structure of the magnetically active regions by examining the local dispersion relation of the p-modes (Ring-diagram analysis) and the travel times of p-modes (time-distance technique). Such studies are crucial as they enable us to estimate the effects that contaminate the sound speeds near the surface of the Sun.

An important although debatable, issue in local helioseismology of magnetically active regions, is the possible influence of surface effects in the interpretation and modelling of helioseismic signatures within sunspots (for contrasting views, see Braun \& Birch 2006; and Zhao \& Kosovichev 2006, and references cited therein). The possibility of unresolved surface perturbations is a plausible reason why some theoretical results are difficult to interpret (e.g. Fan et al. 1995 and Gordovskyy \& Jain 2007b). Yet surface effects not included in the inversion routines affect the overall accuracy of the parameters of the solar interior. Thus, the forward models such as the one proposed here, are potentially critical in helping to establish the existence and importance of surface magnetic effects i.e. forward models may be used to probe the possible existence of perturbations not resolvable and used by current inversions in helioseismology. 
Another important issue regarding the surface effects on acoustic waves is whether the amplitudes and frequencies of these waves are modified due to the localised perturbation in the atmospheric parameters of the magnetic region or due to magnetic field directly. Gordovskyy \& Jain (2007a,b) investigated this aspect by investigating the scattering of p-modes by a thin flux tube in a stratified atmosphere. They found that when a pure acoustic approach is considered where a local variation in pressure, density and sound speed is assumed to be induced by a magnetic field without considering magnetic field explicitly, the phase shift as a function of horizontal wavenumber $k$ is negative where as considering magnetic field explicitly yields positive phase shifts. In this paper we will consider the amplitude modulation by a localised surface magnetic field the measurement of which will indicate the non-intrusive probe of the magnetic field.

\section{The model and the governing equations}

\subsection{Description of the fluxtube model near the surface}

In the solar photosphere, the pressure and density change with depth (or height) but we are interested in the localised effects of surface magnetic fields so we will assume equilibrium density and pressure to be adiabatic and we will also ignore gravity. The basic state $(\boldsymbol{v}, p, \rho, \boldsymbol{B})$ of the fluid is considered to be that of rest. Thus, the linearized equation of ideal MHD may be considered as:

$$
\begin{aligned}
& \frac{\partial \rho^{\prime}}{\partial t}+\rho \nabla \cdot \boldsymbol{v}^{\prime}=0 \\
& \rho \frac{\partial \boldsymbol{v}^{\prime}}{\partial t}+\nabla p^{\prime}-\frac{1}{\mu}\left\{\left[(\nabla \times \boldsymbol{B}) \times \boldsymbol{B}^{\prime}\right]+\left[\left(\nabla \times \boldsymbol{B}^{\prime}\right) \times \boldsymbol{B}\right]\right\}=0 \\
& p^{\prime}=c_{\mathrm{s}}^{2} \rho^{\prime} \boldsymbol{\partial}^{\prime}-\nabla \times\left(\boldsymbol{v}^{\prime} \times \boldsymbol{B}\right)=0 ; \\
& \frac{\nabla}{\partial t}-\boldsymbol{B}^{\prime}=0,
\end{aligned}
$$

where $p^{\prime}, \rho^{\prime}, \boldsymbol{v}^{\prime}$ and $\boldsymbol{B}^{\prime}$ are the perturbations in pressure, density, velocity and magnetic induction due to the sound wave; $c_{\mathrm{s}}^{2}=\frac{\gamma p}{\rho}$ is the velocity of sound in the compressible fluid with $\gamma$ as the ratio of specific heats.

Taking $\mathrm{i} \omega$ for time derivatives of $p^{\prime}, \rho^{\prime}, \boldsymbol{v}^{\prime}$ and $\boldsymbol{B}^{\prime}$ in Eqs. (1)-(4) and eliminating $\rho^{\prime}$ and $\boldsymbol{B}^{\prime}$ from the resulting equations yields an inhomogeneous wave equation for $\boldsymbol{v}^{\prime}$ as follows:

$$
\frac{1}{c_{\mathrm{s}}^{2}} \frac{\partial^{2} \boldsymbol{v}^{\prime}}{\partial t^{2}}-\nabla\left(\nabla \cdot \boldsymbol{v}^{\prime}\right)=\mathcal{L}\left(\boldsymbol{B}, \boldsymbol{v}^{\prime}\right)
$$

where

$$
\begin{aligned}
\mathcal{L}\left(\boldsymbol{B}, \boldsymbol{v}^{\prime}\right)= & \frac{1}{\rho \mu c_{\mathrm{s}}^{2}}\left\{\left[\nabla \times\left(\nabla \times\left(\boldsymbol{v}^{\prime} \times \boldsymbol{B}\right)\right) \times \boldsymbol{B}\right]\right\} \\
& +\left[(\nabla \times \boldsymbol{B}) \times\left(\nabla \times\left(\boldsymbol{v}^{\prime} \times \boldsymbol{B}\right)\right)\right] .
\end{aligned}
$$

The solution of the inhomogeneous wave Eq. (5) can be obtained by first considering the homogeneous problem and then the convolution of the free propagation Green function $G\left(\boldsymbol{r}, t, \boldsymbol{r}^{\prime}, t^{\prime}\right)$ with the term $\mathcal{L}$ (see also King et al. 2003; Pétrélis \& Lund 2003). Thus, using the Born approximation, we have

$\boldsymbol{v}^{\prime}=\boldsymbol{v}_{\mathrm{i}}+\boldsymbol{v}_{\mathrm{s}}$,

with

$\boldsymbol{v}_{\mathrm{s}}=G * \mathcal{L}\left(\boldsymbol{B}, \boldsymbol{v}_{\mathrm{i}}\right)$.

Here $v_{\mathrm{i}}$ is the solution of the homogeneous part of Eq. (5).
The perturbations are assumed to occur at $\left(\boldsymbol{r}^{\prime}, t^{\prime}\right)$ and propagate at $(\boldsymbol{r}, t)$. Thus, in the far-field approach and in the limit $\left\|\boldsymbol{r}-\boldsymbol{r}^{\prime}\right\| \gg\left\|\boldsymbol{r}^{\prime}\right\|$ (see review by Lund 2002; Pétrélis \& Lund 2003)

$$
\begin{aligned}
G * \mathcal{L}(\boldsymbol{B}, \boldsymbol{v})(\boldsymbol{r}, t)= & \frac{1}{8 \pi^{2} r} \iiint \mathrm{e}^{\mathrm{i} v\left(\|\boldsymbol{r}\| / c_{\mathrm{s}}-t\right)} \mathrm{e}^{\mathrm{i} v\left(t^{\prime}-\boldsymbol{s} \cdot \boldsymbol{r}^{\prime} / c_{\mathrm{s}}\right)} \\
& \times(\mathcal{L}(\boldsymbol{B}, \boldsymbol{v}) \cdot \boldsymbol{s})\left(\boldsymbol{r}^{\prime}, t^{\prime}\right) \mathrm{d} v \mathrm{~d} \boldsymbol{r}^{\prime} \mathrm{d} t^{\prime} \boldsymbol{s},
\end{aligned}
$$

where $s \approx \boldsymbol{r} /\|\boldsymbol{r}\|$

\subsection{Amplitude modulation of acoustic wave by the model flux tube}

This section considers a cartesian coordinate system and assumes the incident wave to be along the $x$-axis with unit vector $i$ and scattered wave in the direction given by the unit vector $s$ $(\cos \theta=s \cdot \boldsymbol{i})$.

For a magnetic field $\left(B_{x}, B_{y}, B_{z}\right)=\left(0,0, B_{0}\right)$, Eq. (8) yields,

$\frac{\left|\boldsymbol{v}_{\mathrm{s}}\right|}{v_{\mathrm{i}}} \propto\left(\frac{v_{\mathrm{A}}^{2}}{c_{\mathrm{S}}^{2}}\right) \frac{1}{\sqrt{\lambda^{3} r}}(1+\cos \theta) \mathcal{F}_{z}\left(\boldsymbol{r}^{\prime}\right)$

where $\mathcal{F}_{z}\left(\boldsymbol{r}^{\prime}\right)=\int \mathrm{e}^{-\mathrm{i} k(\boldsymbol{s}-\boldsymbol{i}) \cdot \boldsymbol{r}^{\prime}} \mathrm{d} \boldsymbol{r}^{\prime} ; \lambda=\frac{\nu}{c_{\mathrm{s}}}$, is the wavelength $(\lambda=$ $2 \pi / k$ for wavenumber $k$ ).

We define a quantity, $\alpha$, the amplitude modulation coefficient, as

$\alpha=\left(\frac{\left|\boldsymbol{v}_{\mathrm{s}}\right|}{v_{\mathrm{i}}}\right)^{2}$.

By measuring $\alpha$ at some angle $\theta$ for a given $\lambda$ and knowing the characteristic size, $R$, of the surface magnetic field region from observations, it is possible to estimate the mean plasma beta, $\beta\left(=\frac{v_{\mathrm{A}}^{2}}{c_{\mathrm{s}}^{2}}\right)$ in the magnetic region at the surface. From (9), it can be seen that there is no scattering for $\theta=\pi$ as there is no $B_{x}$ component.

We will consider two different profiles for $B_{z}$ : uniform vertical field $B_{z}=B_{0}$ and Gaussian field $B_{z}=B_{0} \mathrm{e}^{-\frac{1}{2}\left(\frac{r}{R}\right)^{2}}$. Note that in order to satisfy the solenoidal condition, we should have $z$-dependence in both radial and vertical field components (see for example, Gordovskyy \& Jain 2007b) but here we are only considering $B_{z}$ at $z=0$.

\section{Results and discussion}

Figure 1 illustrates the dependence of the amplitude coefficient, $\alpha$, on distance $r$ away from the magnetic region, for various parameters. The values of $k$ and $R$ are chosen to be $0.09 \mathrm{Mm}^{-1}$ and $10 \mathrm{Mm}$ respectively for the top row and $k=$ $0.045 \mathrm{Mm}^{-1}, R \approx 20 \mathrm{Mm}$ for the bottom row so that the value of $k R(=0.9)$ is the same in all the panels. The solid and dashed curves are for $\beta=0.5$ and 1.0 respectively for the uniform vertical field case. The figure clearly shows that the amplitude of the incident acoustic wave is suppressed $(\alpha<1)$. In fact, the suppression decreases with distance $r$ away from the magnetic field region with significant suppression being seen in the region of closest proximity to the magnetic field. The suppression is also maximum for $\theta=0^{\circ}$ and for larger $\beta$. This is expected as $\alpha$ is proportional to $\cos \theta$ and $\beta$. This figure suggests that if two magnetic regions of equal size have the same magnetic field strength, the modulation could still be different if the plasma $\beta$ of the scattering regions are different. 

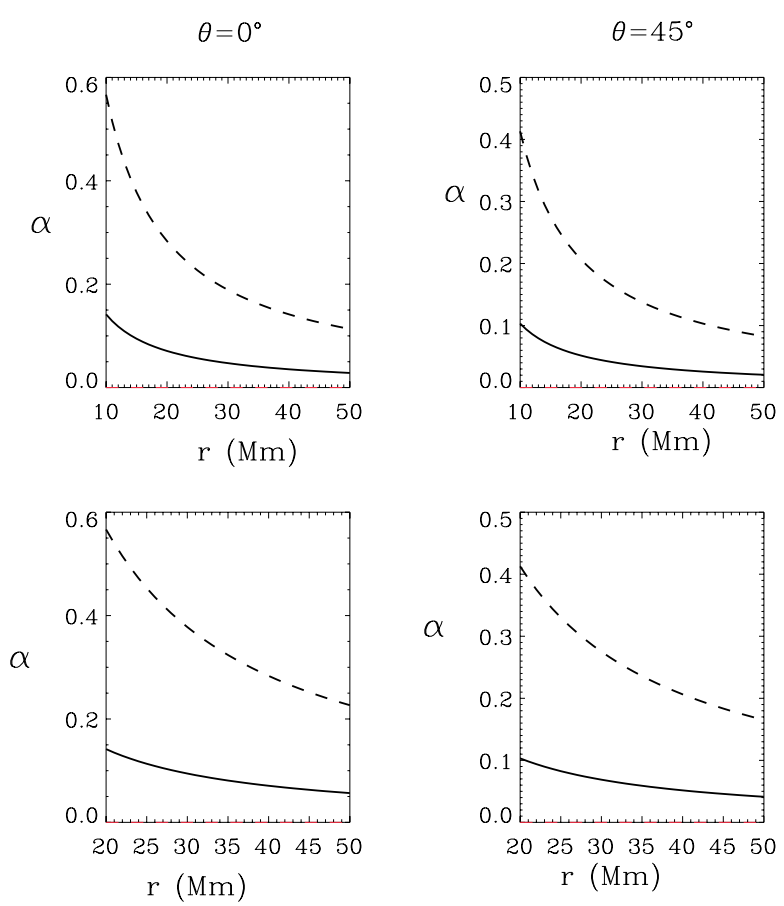

$\beta=0.2$

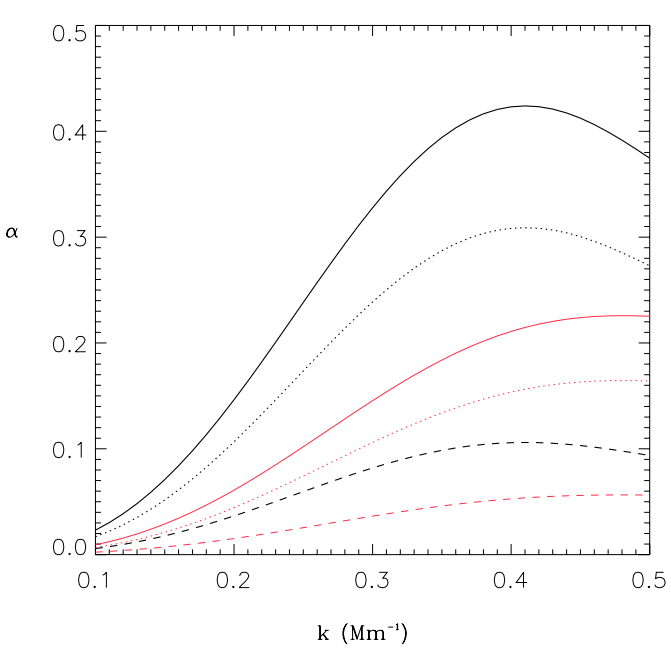

Fig. 2. Absorption coefficient $\alpha$ as a function of wavenumber $k\left(\mathrm{Mm}^{-1}\right)$. The radius $R$ of the magnetic flux tube is assumed to be $5 \mathrm{Mm}$. Various curves denote $\alpha$ at different angles, $\theta$ of scattering: $\theta=0^{\circ}$ (solid), $45^{\circ}$ (dotted), $90^{\circ}$ (dashed) for uniform and Gaussian (coloured or light) fields.

The solar surface contains magnetic fields concentrated in many small-scale regions. It would be interesting to see the variation of $\alpha$ with wavenumber, $k$ for small-scale, weak surface magnetic fields. Figure 2 shows $\alpha$ as a function of $k\left(\mathrm{Mm}^{-1}\right)$ for $\beta=0.2$. The characteristic size, $R$, of the magnetic region is assumed to be $5 \mathrm{Mm}$ and is measured at different angles from the vertical, i.e. $\theta=0^{\circ}$ (solid), $45^{\circ}$ (dotted) and $90^{\circ}$ (dashed). Dark curves are for uniform field and the lighter (or colored) ones are for the Gaussian field case. Significant amplitude modulation is seen in all cases and the relative amplitude of the scattered wave has a maximum with respect to $k$. Figures 1 and 2 clearly shows that the amount of modulation also depends on the direction of measurement and not just the magnitude of the field. This then suggests that if the magnetic fields are inclined, as is the case in magnetic flux tube on the solar surface, the modulation will be a function of line-of-sight. $\theta=90^{\circ}$
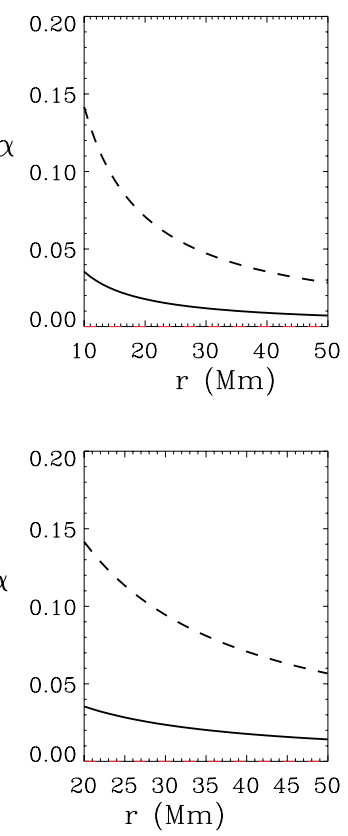

Fig. 1. Absorption coefficient, $\alpha$, as a function of distance $r$ (in $\mathrm{Mm}$ ) for two different sets of values of $k$ and $R$ : the top row has $k=$ $0.09 \mathrm{Mm}^{-1}$ and $R=10 \mathrm{Mm}$ and the bottom row has $k=0.045 \mathrm{Mm}^{-1}$ and $R \approx 20 \mathrm{Mm}$. The various curves are for $\beta=0.5$ (solid) and 1 (dashed).

The relative amplitude of the scattered wave also depends on the characteristic size, $R$, of the magnetic field. It is found that for simple magnetic fields, the amplitude is modulated more by a larger size $(R)$ magnetic flux tube for a given wavelength.

\section{Applicability to observational data}

In order to apply Eq. (10) to a typical solar observation, we use observational data for Active Region (AR) 9026 from Imaging Vector Magnetograph (IVM) at the University of Hawaii Mees Solar Observatory (Mickey et al. 1996). This data provides the magnetic field strength, $|B|$ and the angle of inclination $(\gamma)$ from vertical direction as a function of distance in the sunspot (see for details, Shunkar et al. 2005) at the solar surface. Figure 3 shows this data.

In Fig. 3a we plot $B_{z}=|B| \cos \gamma$ as a function of distance $r$ (in $\mathrm{Mm}$ ) and in Fig. $3 \mathrm{~b} B_{r}=|B| \sin \gamma$. The solid lines in both figures are a Gaussian fit to all the data points to estimate the $B_{z}$ and $B_{r}$ profiles. From $B_{r}(r)$, we calculate $B_{x}=B_{r} \cos \theta$, $B_{y}=B_{r} \sin \theta$, where $\theta$ is the angle between the $x$-axis and the scattering wave. Note that the maximum value of magnetic field strength is about $2.2 \mathrm{kG}$ so for a typical photospheric gas pressure, the value of $\beta$ can be estimated to be 2 . However, one expects the value of $\beta$ to change with distance.

We calculate the coefficient $\alpha$ as given in Eq. (10) with:

$$
\frac{\left|\boldsymbol{v}_{\mathrm{s}}\right|}{v_{\mathrm{i}}} \propto\left(\mu \rho c_{\mathrm{s}}^{2}\right)^{-1} \frac{1}{\lambda^{2} r} \mathcal{T}(\theta)
$$

where $\mathcal{T}(\theta)$ is obtained by Eq. (8) noting that $\boldsymbol{B}=\left(B_{x}, B_{y}, B_{z}\right)$, i.e.

$$
\begin{aligned}
\mathcal{T}(\theta)= & -\frac{\cos 2 \theta(1-\cos \theta)}{2} \int \mathrm{e}^{-\mathrm{i} \frac{k}{2 \pi}(\boldsymbol{s}-\boldsymbol{i}) \cdot \boldsymbol{r}^{\prime}} B_{x}^{2} \mathrm{~d} \boldsymbol{r}^{\prime} \\
& -\frac{\cos 2 \theta(1+\cos \theta)}{2} \int \mathrm{e}^{-\mathrm{i} \frac{k}{2 \pi}(\boldsymbol{s}-\boldsymbol{i}) \cdot \boldsymbol{r}^{\prime}} B_{y}^{2} \mathrm{~d} \boldsymbol{r}^{\prime} \\
& -\frac{(1+\cos \theta)}{2} \int \mathrm{e}^{-\mathrm{i} \frac{k}{2 \pi}(\boldsymbol{s}-\boldsymbol{i}) \cdot \boldsymbol{r}^{\prime}} B_{z}^{2} \mathrm{~d} \boldsymbol{r}^{\prime} \\
& +\cos \theta \sin 2 \theta \int \mathrm{e}^{-\mathrm{i} \frac{k}{2 \pi}(\boldsymbol{s}-\boldsymbol{i}) \cdot \boldsymbol{r}^{\prime}} B_{x} B_{y} \mathrm{~d} \boldsymbol{r}^{\prime}
\end{aligned}
$$



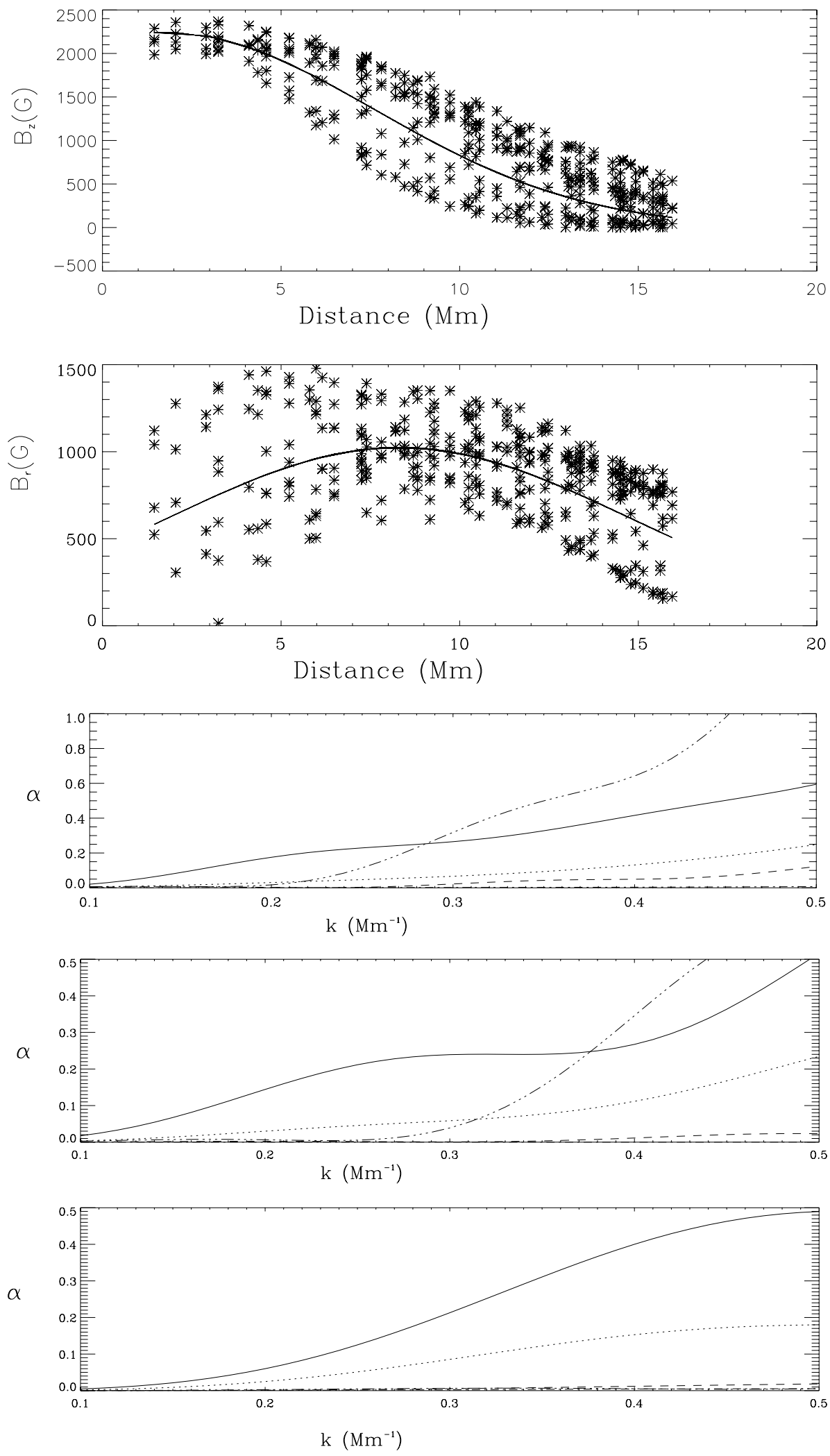

Fig. 3. Estimated $B_{z}$ (top) and $B_{r}$ (bottom) from observational data of AR9026 as a function of distance $(\mathrm{Mm})$. In each panel, the solid curve is a Gaussian fit to the data.
Fig. 4. The calculated value of $\alpha$ from the observational data (see Fig. 3) for $\beta=2$ as a function of wavenumber $k\left(\mathrm{Mm}^{-1}\right)$. The various curves are for $\theta=0$ (solid), $45^{\circ}$ (dotted), $90^{\circ}$ (dashed) and $135^{\circ}$ (dash-dot). The top, middle and bottom panels are for the characteristic size, $R=15 \mathrm{Mm}, 10 \mathrm{Mm}$ and $5 \mathrm{Mm}$ respectively.
Note that in general, $B_{x}, B_{y}$ and $B_{z}$ are expressed in terms of $B_{0}$ and thus the amplitude modulation is directly proportional to the ratio of Alfvén to sound speed in Eq. (11). In Fig. 4, we plot $\alpha$ as a function of $k\left(\mathrm{Mm}^{-1}\right)$ for various $\theta$. The three panels show $\alpha$ for the same value of $\beta(=2)$ but for three different characteristic size regions $15 \mathrm{Mm}$ (top), $10 \mathrm{Mm}$ (middle) and $5 \mathrm{Mm}$ (bottom) respectively.
Figures 3 and 4 clearly suggest that different characteristic sizes, $R$, include magnetic fields of different inclinations. Obviously, depending on the magnitudes of $B_{x}, B_{y}$ and $B_{z}$ components, various curves in Fig. 4 show the variation of $\alpha$ according to Eq. (12). Note that the calculated amplitude modulation is only an approximation here because $\beta(=2)$ is assumed constant throughout the magnetic region. Although caution is required in 

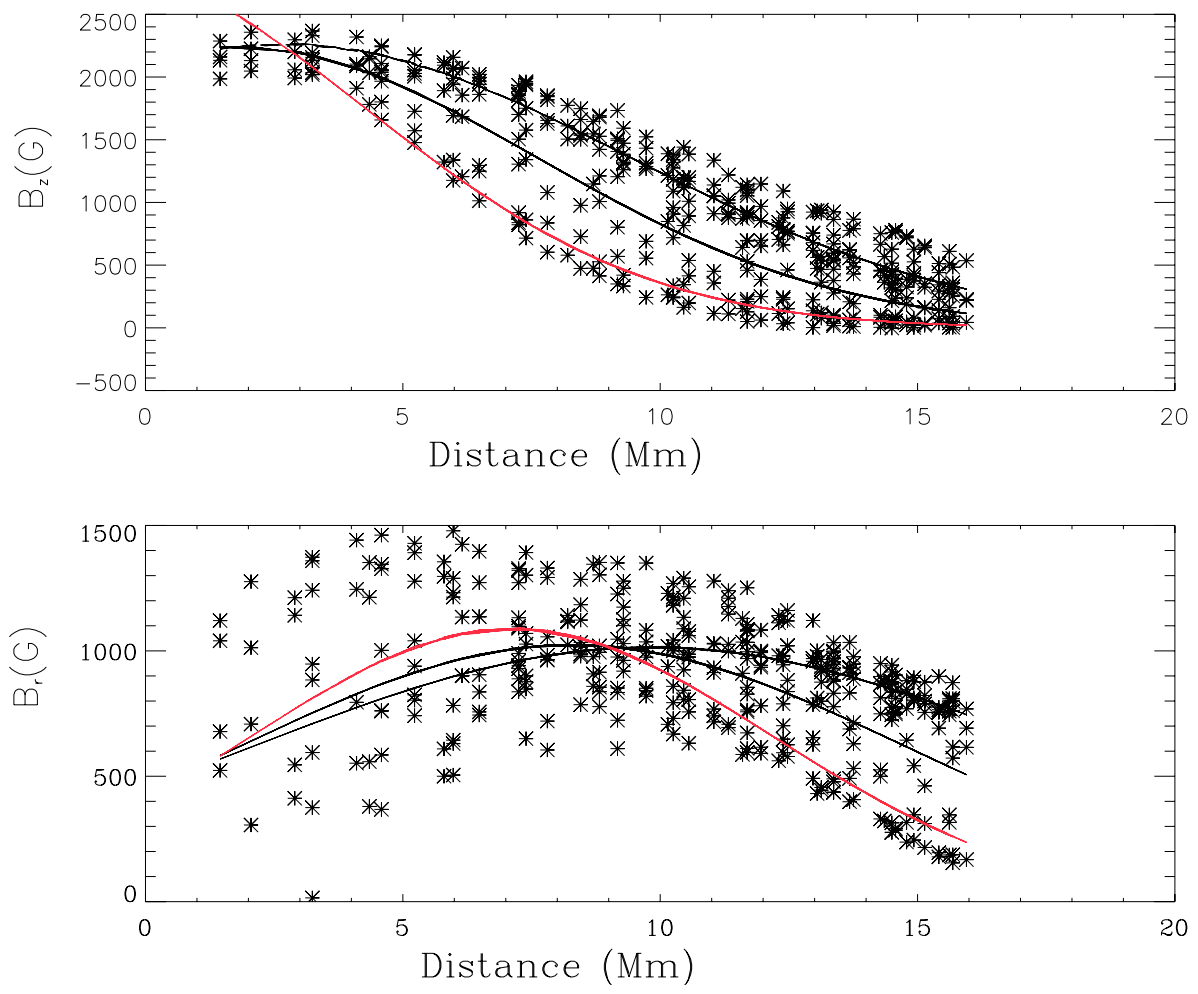

Fig. 5. Estimated $B_{z}$ (top) and $B_{r}$ (bottom) from observational data as a function of distance (Mm). In the top panel, the middle solid curve is a Gaussian fit to the data as shown in Fig. 3a while the other two curves in this panel show the Gaussian fits after the data points are separated according to whether $B_{z}$ is greater than or less than the middle (average) curve. The corresponding $B_{r}$ are shown in the bottom panel. interpreting the curves (since the far-field approach and the Born Approximation are used), it can be seen clearly that for a given wavelength, the amplitude modulation is a function of the scattering angle.

Careful investigation of Fig. 3 suggests that the data points may belong to more than one population in this active region (e.g. different magnetic structures) and thus, $B_{z}$ and $B_{r}$ (and the corresponding values of $B_{x}$ and $B_{y}$ ) can be fitted with separate Gaussian profiles. Populations are separated according to whether $B_{z}$ is greater or less than the Gaussian curve of Fig. 3a. Figure 5a shows this. The corresponding $B_{r}$ profiles are shown in Fig. 5b. We expect the magnitude of $\alpha$ to change slightly as a result of different Gaussian curves but the overall $\theta$ dependence is not expected to vary significantly. In Fig. 6, we consider $\beta=2$, $r=15 \mathrm{Mm}$ and $k=0.3 \mathrm{Mm}^{-1}$ and plot $\alpha$ for various $\theta$ for the three fitted Gaussian profiles.

It is interesting to note that although Fig. 2 (see colored or light curves for the Gaussian profile) and the bottom panel of Fig. 4 are for $R=5 \mathrm{Mm}$, the dependence of $\alpha$ on $\beta$ and $k$ is different in the two cases because in Fig. 4, we also have contributions from $B_{x}$ and $B_{y}$ terms in $\mathcal{T}(\theta)$. In Fig. 7, we plot the coefficients of $B_{x}, B_{y}$ and $B_{z}$ in the expression of $\mathcal{T}(\theta)$ as a function of $\theta$ which clearly shows the contribution of different components of magnetic field at various theta.

\section{Conclusion}

As mentioned in the "Introduction" section, amplitude modulation of solar p-modes by magnetic fields has been investigated by many. Sunspots and Plages show that the $\mathrm{p}$ mode amplitudes are modulated. Although this modulation is present in wider regions around strong magnetic fields, the role of factors other than the local magnetic field strength was not clear.

We have calculated the amplitude modulation of acoustic waves scattered by a localised magnetic field. We considered the direct effect of magnetic field on the amplitudes of acoustic

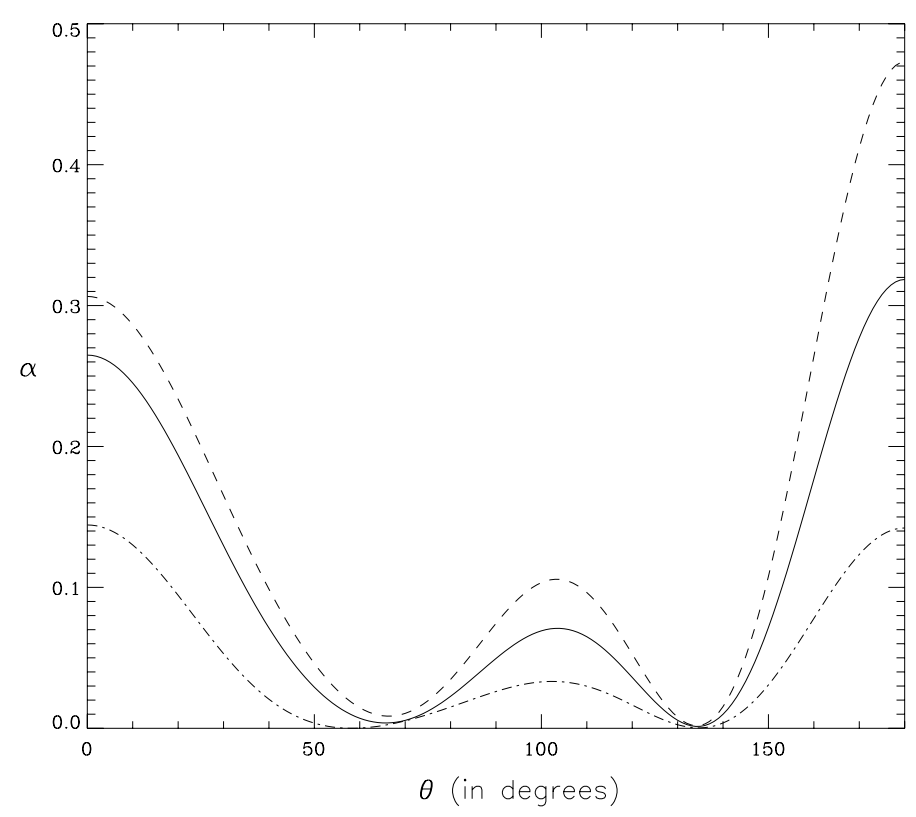

Fig. 6. Amplitude modulation, $\alpha$, as a function of $\theta$ (in degrees) for the three fitted profiles of $B_{z}$ and $B_{r}$ shown in Fig. 5; $\alpha$ calculated from top profile is indicated by the dashed line while the middle and bottom profiles are shown by solid and dot-dashed lines respectively. The characteristic region size of $R=15 \mathrm{Mm}$ and $k=0.3 \mathrm{Mm}^{-1}$ is assumed in all cases.

wave, ignoring the changes in the thermodynamic properties of the plasma due to the presence of a magnetic flux tube. Therefore, ideally measuring such amplitudes in the vicinity of a strong magnetic region is a non-intrusive probe of the magnetic field, although it should be noted that the contributions due to the above two effects, whether magnetic fields or thermodynamic parameter (e.g. temperature, density etc.) variation due to the presence of magnetic field structure, are difficult to distangle 


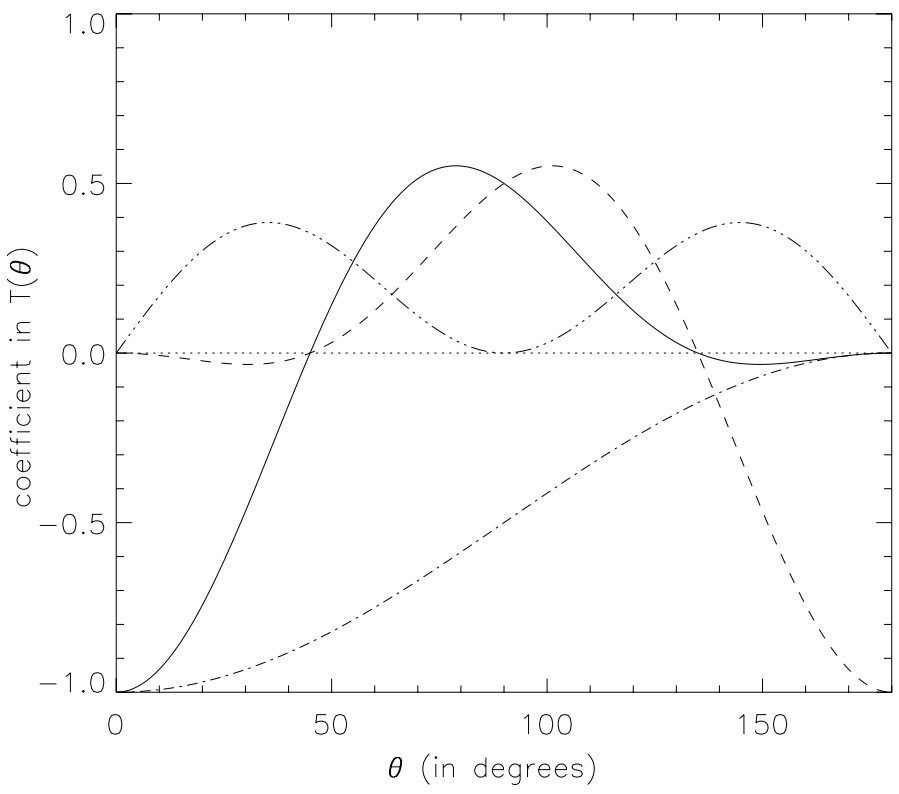

Fig. 7. Coefficients in, $\mathcal{T}(\theta)$, as a function of $\theta$. The dashed, solid, dashdot and dash-dot-dot-dot lines indicate the coefficients of $B_{x}^{2}, B_{y}^{2}, B_{z}^{2}$ and $B_{x} B_{y}$ terms (see Eq. (12)).

in real solar surface observations without further progress in theory (see e.g. Jain et al. 1996; Dzeimbowski et al. 2000; Antia et al. 2001). However, as was noted by Hindman et al. (1997) (see also, Braun \& Birch 2006), in order for the equivalence of magnetic field and thermodynamic changes, the sound speed would need to be a function of wavenumber and frequency which is clearly absurd. Keeping this in mind, it is shown that when direct effect of magnetic field structures is considered, the amplitude modulation depends on the plasma beta, the angle of measurement of scattered wave, the wavenumber and the distance from the scattering region. Since the solar magnetic structures generally have an inclined but non-symmetric fields, the amplitude modulation will be a complicated function of $r$ and $\theta$. Nonetheless, this study shows that $\alpha$ is a function of the direction of the line-of-sight measurement. One of the important consequences of the present work is that it suggests that the acoustic wave information present in the Doppler velocity signals is different in magnetic structure than in the non-magnetic regions due to the interaction of plasma velocity with the surface magnetic fields (see Eq. (5)) but since the temperature and density are not altered between the magnetic and non-magnetic regions, this does not affect the continuum intensity or line-ofdepth data. Thus, one has to be careful in interpreting the analyses of Dopplergram observations (see also, Zhao \& Kosovichev 2006). Further theoretical work is underway for a better understanding of this aspect.

In the present paper, we have used the Born Approximation (BA) to solve the inhomogeneous wave equation. Strictly speaking, BA is only valid for $k R<1$. However, the comparison of Ray approximation and BA by Birch et al. (2001) suggests that the two approximation match well even for $k R \sim 1$. In order to use first order BA, we assumed that the scattering region has to be much smaller i.e. the magnetic field vanishes faster than $r^{-1}$. It is possible that these assumptions are not reasonable for many solar surface magnetic fields. A fully numerical approach may be required to calculate $\alpha$ for acoustic waves of smaller wavelength and their interaction with magnetic flux tubes of larger radii embedded in a "realistic" stratified atmosphere. This is the subject of a future paper (Gordovskyy and Jain, in preparation).

Acknowledgements. R.J. would like to thank F. Pétrélis for many useful discussions and H. Shunker for providing the observational data. I am grateful to the referee for a very careful review of this paper. This work is supported by the Engineering and Physical Sciences Research Council, grant EP/C548795/1.

\section{References}

Abdelatif, T. E., Lites, B. W., \& Thomas, J. H. 1986, ApJ, 311, 1015 Antia, H. M., Basu, S., Hill, F., et al. 2001, Mon. Not. R. Soc., 327, 1029 Birch, A. C., Kosovichev, A. G., Price, G. H., \& Schlottmann, R. B. 2001, ApJ, 561, L229

Braun, D. C. 1995 , ApJ, 451, 859

Braun, D. C., \& Birch, A. C. 2006, ApJ, 647, L187

Braun, D. C., Duvall, T. L., \& Labonte, B. J. 1987, ApJ, 319, L27

Braun, D. C., Duvall, T. L., \& Labonte, B. J. 1988, ApJ, 335, 1015

Cally, P. S., Crouch, A. D., \& Braun, D. C. 2003, MNRAS, 346, 381

Christensen-Dalsgaard, J. 2002, Rev. Mod. Phys., 74, 1073

Duvall Jr, T. L., Jefferies, S. M., Harvey, J. W., \& Pomerantz, M. A. 1993, Nature, 362,430

Dziembowski, W. A., Goode, P. R., Kosovichev, A. G., \& Schou, J. 2000, ApJ, 537,1026

Fan, Y., Braun, D. C., \& Chou, D. Y. 1995, ApJ, 451, 877

Gizon, L., \& Birch, A. C. 2005, Living Rev. Solar Phys., 2 [http: //www. livingreviews.org/lrsp-2005-6]

Goldreich, P., Murray, N., Willette, G., \& Kumar, P. 1991, ApJ, 370, 752

Gordovskyy, M., \& Jain, R. 2007a, Astr. Nachr., accepted

Gordovskyy, M., \& Jain, R. 2007b, ApJ, accepted

Hill, F. 1988, ApJ, 333, 996

Hindman, B. W., \& Brown, T. M. 1998, ApJ, 504, 1029

Hindman, B. W., Jain, R., \& Zweibel, E. G. 1997, ApJ, 476, 392

Howe, R., Komm, R. W., \& Hill, F. 1999, ApJ, 524, 1084

Jain, R., \& Haber, D. 2002, A\&A, 387, 1092

Jain, R., \& Roberts, B. 1993, ApJ, 414, 898

Jain, R., \& Roberts, B. 1994, Sol. Phys., 152, 261

Jain, R., Hindman, B. W., \& Zweibel, E. G. 1996, ApJ, 464, 476

King, A. C., Billigham, J., \& Otto, S. R. 2003, Differential Equations book (Cambridge University Press)

Libbrecht, K. G., \& Woodard, M. F. 1990, Nature, 345, 779

Lund, F. 2002, in Sound-Flow interaction, Lect. Notes Phys., 586 (SpringerVerlag)

Mickey, D. L., Canfield, R. C., Labonte, B. J., et al. 1996, Sol. Phys., 168, 229

Nicholas, C. J., Thompson, M. J., \& Rajaguru, S. P. 2004, Sol. Phys., 225, 213

Pétrélis, F., \& Lund, F. 2003, Eur. Phys. J. B, 35, 291

Schunker, H., Braun, D. C., Cally, P. S., \& Lindsey, C. 2005, ApJ, 621, L149

Thomas, J. H., \& Stanchfield, D. C. H. II 2000, ApJ, 537, 1086

Woodard, M. F., \& Noyes, R. W. 1985, Nature, 318, 449

Zhao, J., \& Kosovichev, A. G. 2006, ApJ, 643, 1317 